\title{
Identificação do Estilo de Aprendizagem utilizando o Modelo LV como auxílio para personalização de Sistemas Tutores Inteligentes
}

\author{
Edilaine S. Oliveira ${ }^{1}$, Gilvandenys L. Sales ${ }^{1}$, Amauri H. Souza ${ }^{1}$, Ramires N. Moreira ${ }^{2}$ \\ ${ }^{1}$ Programa de Pós-Graduação em Ciência da Computação - PPGCC \\ Instituto Federal de Educação, Ciência e Tecnologia do Ceará - \\ Av. Treze de Maio, 2081 - Benfica - 60.040-215 - Fortaleza - CE - Brasil \\ ${ }^{2}$ Departamento de Engenharia de Telecomunicações \\ Instituto Federal de Educação, Ciência e Tecnologia do Ceará - \\ Av. Treze de Maio, 2081 - Benfica - 60.040-215 - Fortaleza - CE - Brasil
}

\{edilaine.santiago9, denyssales, amauri01,ramires.nas\}@gmail.com

\begin{abstract}
The Learning Styles (LS) identification seeks to provide assistance in adaptive systems based on each student's preferences, as well as contributing to recommendation systems and intelligent tutors systems. This research proposes to classify learning profiles from the records of the students' activities, generated by the Learning Vectors Model (LV), within a Learning Management System (LMS). The Felder and Silverman model was used as the basis, initially in the processing dimension. Different machine learning techniques were implemented and compared to perform this classification. As a result, we obtained a maximum accuracy rate of $72.50 \%$ with the KNN algorithm using the LV attributes and $69.99 \%$ with the SVM using attributes chosen from the literature.
\end{abstract}

Resumo. A identificação de Estilos de Aprendizagem (EA) busca fornecer auxílio em sistemas adaptativos baseados nas preferências de cada aluno, assim como pode contribuir em sistemas de recomendação e sistemas tutores inteligentes. Esta pesquisa propõe classificar perfis de aprendizagem a partir dos registros das atividades dos alunos, gerados pelo Modelo Learning Vectors (LV), dentro de um Ambiente Virtual de Aprendizagem (AVA). O modelo de Felder e Silverman foi utilizado como base, inicialmente na dimensão processamento. Diferentes técnicas de aprendizado de máquina foram implementadas e comparadas para realizar esta classificação. Como resultado, obteve-se uma taxa de acurácia máxima de 72,50\% com o algoritmo KNN usando os atributos do LV e 69,99\% com o SVM usando atributos escolhidos com base na literatura.

\section{Introdução}

Uma das desvantagens dos Ambientes Virtuais de Aprendizagem (AVA) é que estes abordam o conteúdo de maneira única para todos os alunos. O uso da Inteligência Artificial na Educação (IA-ED) pode tanto fornecer conteúdo, bem como coletar informações relevantes para o processo de adaptação em sistemas de aprendizagem [Wong and Looi 2012]. 
VII Congresso Brasileiro de Informática na Educação (CBIE 2018)

Anais do XXIX Simpósio Brasileiro de Informática na Educação (SBIE 2018)

Assim, a teoria de Estilos de Aprendizagem possibilita ampliar as formas de aprender, de acordo com as competências e habilidades pessoais do indivíduo, auxiliando na construção de sistemas tutores inteligentes [Graf et al. 2009].

O objetivo deste trabalho é aplicar diferentes algoritmos de classificação para identificar de forma automática o Estilo de Aprendizagem dos alunos, com base no modelo de Felder e Silverman (FSLSM) [Felder and Silverman 1988]. Esta classificação utiliza dados gerados pelas interações nas ferramentas de aprendizagem (fórum, tarefa, quiz, wiki) e dados gerados pelos indicadores quali-quantitativos do modelo de avaliação Learning Vectors (LV) [Sales 2010], dentro do ambiente virtual Moodle (Modular Object-Oriented Dynamic Learning Environment).

\section{Fundamenta Teórica}

\subsection{Modelo Learning Vectors (LV)}

O Modelo Learning Vectors (LV), proposto por [Sales 2010], se destaca por ser um modelo de suporte e gerenciamento à avaliação formativa. Este modelo foi concebido para automatizar o acompanhamento do desempenho qualitativo/quantitativo de alunos na EaD dentro de um AVA. O LV atua sobre a interação entre os alunos e suas ações sobre as ferramentas do ambiente de aprendizagem, como fórum, tarefa e wiki. A avaliação é feita através de menções qualitativas: Muito Bom, Bom, Regular, Fraco, Não Satisfatório e Neutro, mediadas por ícones. A razão entre os resultados positivos e negativos das contribuições dos alunos nas atividades, compõem uma métrica pedagógica não-linear denominada Fator $\beta$. Essa métrica se relaciona com a natureza qualitativa do modelo de avaliação LV, considerando o desempenho do indivíduo em cada atividade.

\subsection{Estilos de Aprendizagem de Felder e Silverman}

O modelo de Estilos de Aprendizagem de Felder e Silverman (FSLSM), apresenta a forma de como os indivíduos preferem processar, perceber, captar e organizar a informação [Felder and Silverman 1988]. Neste modelo os alunos são classificados em quatro dimensões diferentes: ativo/reflexivo, sensorial/intuitivo, visual/verbal, sequencial/global. O uso frequente deste modelo se dá ao fato dele ter sido baseado em extensa experimentação, na qual se validou todas as dimensões em uma população de alunos de engenharia [Dorça 2012]. Para detectar esses perfis, foi desenvolvido o questionário Index Learning Styles (ILS) que contém 44 questões objetivas, no qual para cada dimensão existem 11 questões equivalentes, cada uma com duas opções de escolha [Felder and Soloman 1997].

\section{Trabalhos Relacionados}

Em seu trabalho, [Graf et al. 2009] citam que o uso de EAs ajuda a melhorar a eficiência da adaptatividade dos sistemas de recomendação, além de trazer modelos de aprendizagem mais abrangentes. Outra pesquisa que também emprega o modelo de Felder e Silverman é a de [Hung et al. 2016], no qual nesse estudo utilizou-se dados gerados por um ambiente virtual gamificado, para a classificação dos perfis de alunos.

O Modelo LV vem sendo utilizado em diversas aplicações, tanto na área da educação como na área corporativa e tem apresentado resultados satisfatórios. No trabalho de [G. Cacais and L. Sales 2017], o modelo foi utilizado em um sistema colabora- 
VII Congresso Brasileiro de Informática na Educação (CBIE 2018)

Anais do XXIX Simpósio Brasileiro de Informática na Educação (SBIE 2018)

tivo gamificado para avaliação de desempenho corporativo, denominado Process Planning and Institutional Evaluation (PIPA). O Modelo LV auxiliou no acompanhamento da progressão dos profissionais, possibilitando um melhor feedback pelos seus supervisores. Outro recente trabalho que aborda o Modelo LV foi o de [Albuquerque et al. 2017]. Nesta pesquisa foi proposto um modelo utilizando lógica Fuzzy para avaliar a presencialidade de alunos dentro de um fórum LV. O resultado do artigo mostrou que o modelo foi capaz de mensurar a presencialidade do aluno com perspectivas de ser aplicado a outras ferramentas de avaliação presentes nos AVAs.

\section{Metodologia}

Para a realização desta pesquisa foram utilizados dados referentes à primeira disciplina de dois cursos EaD de pós-graduação. Estes dois cursos foram selecionados pois utilizam o Modelo LV como método de avaliação. De forma a coletar os estilos de aprendizagem dos alunos, o questionário ILS foi disponibilizado através de uma plataforma web e aplicado aos alunos do curso avaliado. A resposta de cada aluno foi registrada e utilizada para rotular o conjunto de atributos de acordo com o perfil atribuído pelo questionário. Os atributos coletados da base de dados do Moodle foram escolhidos com base no trabalho de [Graf et al. 2009], que apresenta quais atributos são relevantes na identificação dos perfis.

Outros atributos referentes ao Modelo LV também foram extraídos. Ao todo foram extraídos 21 atributos (Figura1), referentes aos $\log s$ gerados pelas interações dos alunos a partir de várias ferramentas educacionais disponíveis no ambiente virtual, como: fórum, quiz, glossário, tarefa, wiki e outras, capazes de descrever as preferências de cada aluno. De todos atributos extraídos, apenas seis foram selecionados para a dimensão de processamento avaliada neste artigo, onde estes contribuem para a identificação do perfil ativo/reflexivo: num_avaliacao_bom, fator_beta_tarefalv, fator_beta_quizlv, total_add_glossario, total_arq_enviado_tarefa, tota_tentativas_quiz. Esses seis atributos escolhidos foram selecionados a partir de um método de seleção de atributos, nomeado como Recursive Feature Elimination (RFE). Este método de seleção é capaz de calcular a combinação de atributos que contribuem para a previsão da classe, aumentando a precisão e diminuindo o tempo de processamento.

\begin{tabular}{ll}
\hline Recursos Educacionais & \multicolumn{1}{c}{ Dados Coletados } \\
\hline Fórum LV & view_forum, post_forum \\
Glossário & add_gloss, view_gloss \\
Tarefa LV & arq_env_tarefa, view_tarefa \\
Quiz LV & review_quiz, media_tempo_quiz, attempt_quiz, \\
Material da Disciplina & view_conteudo \\
Visão Geral Disciplina & view_visaogeral \\
& num_avaliacao_muitobom, num_avaliacao_regular, \\
& num_avaliacao_fraco, num_avaliacao_bom, \\
Modelo LV & num_avaliacao_naosatisfatorio,fator_beta_forumlv, \\
& fator_beta_tarefalv, fator_beta_wikilv, fator_beta_quizlv, \\
& fator_beta_disciplina \\
\hline
\end{tabular}

Figura 1. Recursos e atributos avaliados 
VII Congresso Brasileiro de Informática na Educação (CBIE 2018)

Anais do XXIX Simpósio Brasileiro de Informática na Educação (SBIE 2018)

Foram criadas duas bases de dados, uma apenas com atributos referentes ao Modelo LV, onde os selecionados foram: num_avaliacao_bom, fator_beta_tarefalv, fator_beta_quizlv. Já na outra base, somente os atributos usados na literatura: total_add_glossario, total_arq_enviado_tarefa, tota_tentativas_quiz, de forma a comparar os resultados das taxas de acertos de cada uma. Utilizou-se a linguagem Python com auxílio da biblioteca scikit-learn [Pedregosa et al. 2011] para uso dos classificadores e a biblioteca Pandas para análise de dados. Neste primeiro experimento, as bases criadas são referentes aos dados de 30 alunos. Os classificadores foram treinados apenas na dimensão de processamento do modelo FSLSM, que classifica o indivíduo em ativo ou reflexivo. Os classificadores k-Nearest Neighbor (KNN), Multinomial Naive Bayes, Perceptron Multicamadas (MLP) e Support Vector Machine (SVM) foram utilizados. No treinamento dos classificadores foi aplicada a técnica de validação cruzada k-fold, com k=10 e Grid Search para escolha dos melhores parâmetros de cada algoritmo.

\section{Análise e Discussão dos Resultados}

Pode-se observar que a média da acurácia nos dois conjuntos de dados foram aproximadas, indicando a possibilidade dos atributos do Modelo LV identificarem os perfis dos alunos na dimensão processamento do modelo FSLSM. Os resultados referentes às 30 execuções nos dois datasets estão expostos a seguir nas Tabelas 1 e 2. Na classificação com o Modelo LV, o algoritmo KNN conseguiu obter uma acurácia de 72,50\%. Já nos resultados obtidos com atributos da literatura, o SVM apresentou os melhores resultados. Este algoritmo atingiu uma taxa de acurácia de quase $70 \%$ e F1 score de 0.76 (Tabela 1).

Tabela 1. Classificação Atributos Literatura - Dimensão Processamento

\begin{tabular}{lllll}
\hline Algoritmo & Acurácia & Precisão & Recall & F1 Score \\
\hline KNN & $62,50 \%$ & 0.50 & 0.64 & 0.55 \\
MultinomialNaiveBayes & $67,50 \%$ & 0.56 & 0.69 & 0.61 \\
MLP & $59,99 \%$ & 0.48 & 0.71 & 0.55 \\
SVM & $69,99 \%$ & 0.68 & 0.89 & 0.76 \\
\hline
\end{tabular}

Tabela 2. Classificação Atributos Modelo LV - Dimensão Processamento

\begin{tabular}{lllll}
\hline Algoritmo & Acurácia & Precisão & Recall & F1 Score \\
\hline KNN & $72,50 \%$ & 0.63 & 0.69 & 0.64 \\
MultinomialNaiveBayes & $42,50 \%$ & 0.25 & 0.34 & 0.28 \\
MLP & $64,00 \%$ & 0.62 & 0.72 & 0.61 \\
SVM & $67,50 \%$ & 0.56 & 0.55 & 0.53 \\
\hline
\end{tabular}

Na classificação com os atributos do Modelo LV, o algoritmo que obteve melhor performance foi KNN, no qual o valor de K escolhido no Grid Search foi igual a 1. A segunda maior acurácia foi do modelo SVM, porém observando as outras métricas, como precisão, recall e F1, percebe-se que o modelo do MLP se sobressai. A média da taxa de acerto no primeiro dataset (Tabela 1) foi de $64,99 \%$, já no segundo (Tabela 2) a média foi de $61,62 \%$. 
VII Congresso Brasileiro de Informática na Educação (CBIE 2018)

Anais do XXIX Simpósio Brasileiro de Informática na Educação (SBIE 2018)

\section{Conclusão e Trabalhos Futuros}

Este trabalho apresenta parte de uma pesquisa que ainda está em progresso. A pesquisa contribui para o estado da arte, ao propor a identificação de estilos através da combinação do LV e FSLSM associados à técnicas de classificação. Os resultados preliminares indicam que o Modelo LV também pode ser usado para identificar o perfil do aluno, pois apresentou acurácias semelhantes aos testes realizados com atributos da literatura e em alguns casos apresentou resultados ainda melhores. Como trabalho futuro pretende-se utilizar a técnica de Over-Sampling de forma a tratar o problema do desbalanceamento de classes nas outras dimensões, a fim de conseguir um maior número de dados para melhor avaliar o desempenho dos classificadores e testar todas as dimensões do modelo FSLSM. Pretende-se ainda, aplicar este modelo de classificação de EA num Ambiente Virtual de Aprendizagem para personalização deste ambiente.

\section{Referências}

Albuquerque, M. C. N., Sales, G. L., Filho, P. P. R., and de Sá Medeiros, C. M. (2017). Avaliação da presencialidade em um fórum lv utilizando lógica fuzzy. XXVIII Simpósio Brasileiro de Informática na Educação (SBIE 2017).

Dorça, F. A. (2012). Uma abordagem estocástica baseada em aprendizagem por reforço para modelagem automática e dinâmica de estilos de aprendizagem de estudantes em sistemas adaptativos e inteligentes para educação a distância. $\mathrm{PhD}$ thesis, Universidade Federal de Uberlândia, Uberlândia.

Felder, R. M. and Silverman, L. K. (1988). Learning and teaching styles in engineering education. ENGINEERING EDUCATION.

Felder, R. M. and Soloman, B. A. (1997). Index of learning styles questionnaire.

G. Cacais, M. and L. Sales, G. (2017). A collaborative system for corporate performance evaluation using gamification and the learning vectors model. In 2017 12th International Conference for Internet Technology and Secured Transactions (ICITST), pages 484-489.

Graf, S., Kinshuk, and Liu, T.-C. (2009). Supporting teachers in identifying students' learning styles in learning management systems: An automatic student modelling approach. Journal of Educational Technology and Society, 12(4):3-14.

Hung, Y. H., Chang, R. I., and Lin, C. F. (2016). Hybrid learning style identification and developing adaptive problem-solving learning activities. Computers in Human Behavior, 55:552 - 561 .

Pedregosa, F., Varoquaux, G., Gramfort, A., Michel, V., Thirion, B., Grisel, O., Blondel, M., Prettenhofer, P., Weiss, R., Dubourg, V., Vanderplas, J., Passos, A., Cournapeau, D., Brucher, M., Perrot, M., and Duchesnay, E. (2011). Scikit-learn: Machine learning in Python. Journal of Machine Learning Research, 12:2825-2830.

Sales, G. L. (2010). Learning Vectors (LV): um modelo de avaliação da aprendizagem em EaD online aplicando métricas não-lineares. Tese de doutorado, Universidade Federal do Ceará, Fortaleza, CE, Brasil.

Wong, L.-H. and Looi, C.-K. (2012). Swarm intelligence: new techniques for adaptive systems to provide learning support. Interactive Learning Environments, 20(1):19-40. 